Article

\title{
Combination Synchronization of Three Identical or Different Nonlinear Complex Hyperchaotic Systems
}

\author{
Xiaobing Zhou *, Murong Jiang and Yaqun Huang \\ School of Information Science and Engineering, Yunnan University, Kunming 650091, China \\ * Author to whom correspondence should be addressed; E-Mail: zhouxb.cn@gmail.com.
}

Received: 5 August 2013; in revised form: 3 September 2013 / Accepted: 4 September 2013 /

Published: 10 September 2013

\begin{abstract}
In this paper, we investigate the combination synchronization of three nonlinear complex hyperchaotic systems: the complex hyperchaotic Lorenz system, the complex hyperchaotic Chen system and the complex hyperchaotic Lü system. Based on the Lyapunov stability theory, corresponding controllers to achieve combination synchronization among three identical or different nonlinear complex hyperchaotic systems are derived, respectively. Numerical simulations are presented to demonstrate the validity and feasibility of the theoretical analysis.
\end{abstract}

Keywords: combination synchronization; Lyapunov stability theory; complex hyperchaotic Lorenz system; complex hyperchaotic Chen system; complex hyperchaotic Lü system

\section{Introduction}

Since Fowler et al. [1], introduced a complex Lorenz model to generalize the real Lorenz model in 1982, complex chaotic and hyperchaotic systems have attracted increasing attention, due to the fact that systems with complex variables can be used to describe the physics of a detuned laser, rotating fluids, disk dynamos, electronic circuits and particle beam dynamics in high energy accelerators [2]. When applying complex systems in communications, the complex variables will double the number of variables and can increase the content and security of the transmitted information. Many complex chaotic and hyperchaotic systems have been proposed ever since the 1980s. In [3], the authors studied the chaotic unstable limit cycles of complex Van der Pol oscillators. The rich dynamics behaviors of the complex Chen and complex Lü systems were investigated in [4]. By adding state feedback controllers to 
their complex chaotic systems, complex hyperchaotic Chen, Lorenz and Lü systems were introduced and studied in [5-7], respectively. The authors [8] constructed a complex nonlinear hyperchaotic system by adding a cross-product nonlinear term to the complex Lorenz system. A complex modified hyperchaotic Lü system [9] was proposed by introducing complex variables to its real counterpart.

In 1990 [10], Pecora and Carroll proposed the drive-response concept for constructing the synchronization of coupled chaotic systems. Over the last two decades, synchronization in chaotic systems has been extensively investigated, due to its potential applications in various fields, such as chemical reactions, biological systems and secure communication. Mahmoud et al. [11] designed an adaptive control scheme to study the complete synchronization of chaotic complex nonlinear systems with uncertain parameters. The authors achieved phase synchronization and antiphase synchronization of two identical hyperchaotic complex nonlinear systems via an active control technique in [12]. Based on passive theory, the authors studied the projective synchronization of hyperchaotic complex nonlinear systems and its application in secure communications [13]. Liu et al. [14] investigated the modified function projective synchronization of general chaotic complex systems described by a unified mathematical expression.

The aforementioned synchronization schemes are based on the usual drive-response synchronization mode, which has one drive system and one response system. Recently, Luo [15] proposed a combination synchronization scheme, which has two drive systems and one response system. This synchronization scheme has advantages over the usual drive-response synchronization, such as being able to provide greater security in secure communication. In secure communication, the transmitted signals can be split into several parts, each part loaded in different drive systems, or can divide time into different intervals, the signals in different intervals being loaded in different drive systems. Thus, the transmitted signals can have stronger anti-attack ability and anti-translated capability than those transmitted by the usual transmission model.

Motivated by the above discussions, this paper aims to study the combination synchronization of three identical or different nonlinear complex hyperchaotic systems. The rest of this paper is organized as follows. Section 2 introduces the scheme of combination synchronization. In Section 3 and Section 4, we investigate combination synchronization among three identical and different complex nonlinear hyperchaotic systems, respectively. Finally, conclusions are given in Section 5.

\section{The Scheme of Combination Synchronization}

Suppose that there are three nonlinear dynamical systems, two drive systems and one response system. The drive systems are given by:

$$
\dot{x}=f(x),
$$

and

$$
\dot{y}=g(y) \text {. }
$$

The response system is described by:

$$
\dot{z}=h(z)+U(x, y, z)
$$


where $x=\left(x_{1}, x_{2}, \ldots, x_{n}\right)^{T}, y=\left(y_{1}, y_{2}, \ldots, y_{n}\right)^{T}, z=\left(z_{1}, z_{2}, \ldots, z_{n}\right)^{T}$ are the state vectors of systems (1), (2) and (3), respectively, $f(\cdot), g(\cdot), h(\cdot): R^{n} \rightarrow R^{n}$ are three continuous vector functions and $U(\cdot): R^{n} \times R^{n} \times R^{n} \rightarrow R^{n}$ is a controller vector, which will be designed.

Definition 1 [15]. For drive systems (1) and (2) and response system (3), they are said to be in combination synchronization if there exists three constant matrices, $A, B, C \in R^{n}$ and $C \neq 0$, such that:

$$
\lim _{t \rightarrow+\infty}\|A x+B y-C z\|=0
$$

where $\|\cdot\|$ represents the matrix norm.

\section{Combination Synchronization among Identical Nonlinear Complex Hyperchaotic Systems}

In this section, we take the complex hyperchaotic Lorenz system [6] as an example to investigate the combination synchronization among three identical systems.

The first drive system is given by:

$$
\left\{\begin{array}{l}
\dot{x}_{11}=\alpha\left(x_{21}-x_{11}\right)+x_{41} \\
\dot{x}_{21}=\gamma x_{11}-x_{21}-x_{11} x_{31} \\
\dot{x}_{31}=\frac{1}{2}\left(\bar{x}_{11} x_{21}+x_{11} \bar{x}_{21}\right)-\beta x_{31}+x_{41} \\
\dot{x}_{41}=\frac{1}{2}\left(\bar{x}_{11} x_{21}+x_{11} \bar{x}_{21}\right)-\sigma x_{41}
\end{array}\right.
$$

and the second drive system is described as follows:

$$
\left\{\begin{array}{l}
\dot{x}_{12}=\alpha\left(x_{22}-x_{12}\right)+x_{42} \\
\dot{x}_{22}=\gamma x_{12}-x_{22}-x_{12} x_{32} \\
\dot{x}_{32}=\frac{1}{2}\left(\bar{x}_{12} x_{22}+x_{12} \bar{x}_{22}\right)-\beta x_{32}+x_{42} \\
\dot{x}_{42}=\frac{1}{2}\left(\bar{x}_{12} x_{22}+x_{12} \bar{x}_{22}\right)-\sigma x_{42} .
\end{array}\right.
$$

The response system takes the following form:

$$
\left\{\begin{array}{l}
\dot{x}_{13}=\alpha\left(x_{23}-x_{13}\right)+x_{43}+U_{1}+i U_{2}, \\
\dot{x}_{23}=\gamma x_{13}-x_{23}-x_{13} x_{33}+U_{3}+i U_{4}, \\
\dot{x}_{33}=\frac{1}{2}\left(\bar{x}_{13} x_{23}+x_{13} \bar{x}_{2}\right)-\beta x_{33}+x_{43}+U_{5}, \\
\dot{x}_{43}=\frac{1}{2}\left(\bar{x}_{13} x_{23}+x_{13} \bar{x}_{23}\right)-\sigma x_{43}+U_{6},
\end{array}\right.
$$

where $\alpha, \beta, \gamma$ and $\sigma$ are positive parameters, $x_{11}=u_{1}+i u_{2}, x_{21}=u_{3}+i u_{4}, x_{12}=v_{1}+i v_{2}, x_{22}=$ $v_{3}+i v_{4}, x_{13}=w_{1}+i w_{2}, x_{23}=w_{3}+i w_{4}$ are complex variables and $i=\sqrt{-1} ; u_{i}, v_{i}, w_{i}(i=1,2,3,4)$, $x_{31}=u_{5}, x_{41}=u_{6}, x_{32}=v_{5}, x_{42}=v_{6}, x_{33}=w_{5}, x_{43}=w_{6}$ are real variables. The overbar represents a complex conjugate function. $U_{1}, U_{2}, U_{3}, U_{4}, U_{5}$ and $U_{6}$ are real controllers to be determined.

For the convenience of our discussions, we assume $A=\operatorname{diag}\left(l_{1}, l_{2}, l_{3}, l_{4}\right), B=$ $\operatorname{diag}\left(m_{1}, m_{2}, m_{3}, m_{4}\right), C=\operatorname{diag}\left(k_{1}, k_{2}, k_{3}, k_{4}\right)$ in our synchronization scheme. 
We define error states between systems (5), (6) and (7) as:

$$
\left\{\begin{aligned}
e_{1}+i e_{2} & =k_{1} x_{13}-l_{1} x_{11}-m_{1} x_{12} \\
e_{3}+i e_{4} & =k_{2} x_{23}-l_{2} x_{21}-m_{2} x_{22} \\
e_{5} & =k_{3} x_{33}-l_{3} x_{31}-m_{3} x_{32} \\
e_{6} & =k_{4} x_{43}-l_{4} x_{41}-m_{4} x_{42}
\end{aligned}\right.
$$

such that:

$$
\left\{\begin{array}{l}
\lim _{t \rightarrow \infty}\left\|k_{1} x_{13}-l_{1} x_{11}-m_{1} x_{12}\right\|=0, \\
\lim _{t \rightarrow \infty}\left\|k_{2} x_{23}-l_{2} x_{21}-m_{2} x_{22}\right\|=0, \\
\lim _{t \rightarrow \infty}\left\|k_{3} x_{33}-l_{3} x_{31}-m_{3} x_{32}\right\|=0, \\
\lim _{t \rightarrow \infty}\left\|k_{4} x_{43}-l_{4} x_{41}-m_{4} x_{42}\right\|=0 .
\end{array}\right.
$$

Thus, we have the following error dynamical system:

$$
\left\{\begin{aligned}
\dot{e}_{1}+i \dot{e}_{2} & =k_{1} \dot{x}_{13}-l_{1} \dot{x}_{11}-m_{1} \dot{x}_{12} \\
\dot{e}_{3}+i \dot{e}_{4} & =k_{2} \dot{x}_{23}-l_{2} \dot{x}_{21}-m_{2} \dot{x}_{22} \\
\dot{e}_{5} & =k_{3} \dot{x}_{33}-l_{3} \dot{x}_{31}-m_{3} \dot{x}_{32} \\
\dot{e}_{6} & =k_{4} \dot{x}_{43}-l_{4} \dot{x}_{41}-m_{4} \dot{x}_{42}
\end{aligned}\right.
$$

Substituting Equations (5)-(7) in Equation (10) and separating the real and imaginary parts yields:

$$
\left\{\begin{aligned}
\dot{e}_{1} & =k_{1}\left[\alpha\left(w_{3}-w_{1}\right)+w_{6}\right]-l_{1}\left[\alpha\left(u_{3}-u_{1}\right)+u_{6}\right]-m_{1}\left[\alpha\left(v_{3}-v_{1}\right)+v_{6}\right]+k_{1} U_{1}, \\
\dot{e}_{2} & =k_{1} \alpha\left(w_{4}-w_{2}\right)-l_{1} \alpha\left(u_{4}-u_{2}\right)-m_{1} \alpha\left(v_{4}-v_{2}\right)+k_{1} U_{2}, \\
\dot{e}_{3} & =k_{2}\left(\gamma w_{1}-w_{3}-w_{1} w_{5}\right)-l_{2}\left(\gamma u_{1}-u_{3}-u_{1} u_{5}\right)-m_{2}\left(\gamma v_{1}-v_{3}-v_{1} v_{5}\right)+k_{2} U_{3}, \\
\dot{e}_{4} & =k_{2}\left(\gamma w_{2}-w_{4}-w_{2} w_{5}\right)-l_{2}\left(\gamma u_{2}-u_{4}-u_{2} u_{5}\right)-m_{2}\left(\gamma v_{2}-v_{4}-v_{2} v_{5}\right)+k_{2} U_{4}, \\
\dot{e}_{5} & =k_{3}\left(w_{1} w_{3}+w_{2} w_{4}-\beta w_{5}+w_{6}\right)-l_{3}\left(u_{1} u_{3}+u_{2} u_{4}-\beta u_{5}+u_{6}\right) \\
& -m_{3}\left(v_{1} v_{3}+v_{2} v_{4}-\beta v_{5}+v_{6}\right)+k_{3} U_{5}, \\
\dot{e}_{6} & =k_{4}\left(w_{1} w_{3}+w_{2} w_{4}-\sigma w_{6}\right)-l_{4}\left(u_{1} u_{3}+u_{2} u_{4}-\sigma u_{6}\right)-m_{4}\left(v_{1} v_{3}+v_{2} v_{4}-\sigma v_{6}\right)+k_{4} U_{6} .
\end{aligned}\right.
$$

Then, we obtain the following results. 
Theorem 1. If the controllers are chosen as follows:

$$
\left\{\begin{aligned}
U_{1}= & -\frac{1}{k_{1}}\left\{\left(k_{1} w_{1}-l_{1} u_{1}-m_{1} v_{1}\right)+\left[k_{1} \alpha\left(w_{3}-w_{1}\right)+k_{1} w_{6}-l_{1} \alpha\left(u_{3}-u_{1}\right)-l_{1} u_{6}\right.\right. \\
& \left.\left.-m_{1} \alpha\left(v_{3}-v_{1}\right)-m_{1} v_{6}\right]-\alpha\left(k_{1} w_{2}-l_{1} u_{2}-m_{1} v_{2}\right)\right\} \\
U_{2}= & -\frac{1}{k_{1}}\left\{\left(k_{1} w_{2}-l_{1} u_{2}-m_{1} v_{2}\right)+\left[k_{1} \alpha\left(w_{4}-w_{2}\right)-l_{1} \alpha\left(u_{4}-u_{2}\right)-m_{1} \alpha\left(v_{4}-v_{2}\right)\right]\right. \\
& \left.+\alpha\left(k_{1} w_{1}-l_{1} u_{1}-m_{1} v_{1}\right)-\gamma\left(k_{2} w_{3}-l_{2} u_{3}-m_{2} v_{3}\right)\right\} \\
U_{3}= & -\frac{1}{k_{2}}\left\{\left(k_{2} w_{3}-l_{2} u_{3}-m_{2} v_{3}\right)+\left[k_{2}\left(\gamma w_{1}-w_{3}-w_{1} w_{5}\right)-l_{2}\left(\gamma u_{1}-u_{3}-u_{1} u_{5}\right)\right.\right. \\
& \left.\left.-m_{2}\left(\gamma v_{1}-v_{3}-v_{1} v_{5}\right)\right]+\gamma\left(k_{1} w_{2}-l_{1} u_{2}-m_{1} v_{2}\right)-\beta\left(k_{2} w_{4}-l_{2} u_{4}-m_{2} v_{4}\right)\right\} \\
U_{4}= & -\frac{1}{k_{2}}\left\{\left(k_{2} w_{4}-l_{2} u_{4}-m_{2} v_{4}\right)+\left[k_{2}\left(\gamma w_{2}-w_{4}-w_{2} w_{5}\right)-l_{2}\left(\gamma u_{2}-u_{4}-u_{2} u_{5}\right)\right.\right. \\
& \left.\left.-m_{2}\left(\gamma v_{2}-v_{4}-v_{2} v_{5}\right)\right]+\beta\left(k_{2} w_{3}-l_{2} u_{3}-m_{2} v_{3}\right)-\sigma\left(k_{3} w_{5}-l_{3} u_{5}-m_{3} v_{5}\right)\right\} \\
U_{5}= & -\frac{1}{k_{3}}\left\{\left(k_{3} w_{5}-l_{3} u_{5}-m_{3} v_{5}\right)+\left[k_{3}\left(w_{1} w_{3}+w_{2} w_{4}-\beta w_{5}+w_{6}\right)-l_{3}\left(u_{1} u_{3}+u_{2} u_{4}-\beta u_{5}+u_{6}\right)\right.\right. \\
& \left.\left.-m_{3}\left(v_{1} v_{3}+v_{2} v_{4}-\beta v_{5}+v_{6}\right)\right]+\sigma\left(k_{2} w_{4}-l_{2} u_{4}-m_{2} v_{4}\right)-\alpha\left(k_{4} w_{6}-l_{4} u_{6}-m_{4} v_{6}\right)\right\} \\
U_{6}= & -\frac{1}{k_{4}}\left\{\left(k_{4} w_{6}-l_{4} u_{6}-m_{4} v_{6}\right)+\left[k_{4}\left(w_{1} w_{3}+w_{2} w_{4}-\sigma w_{6}\right)-l_{4}\left(u_{1} u_{3}+u_{2} u_{4}-\sigma u_{6}\right)\right.\right. \\
& \left.\left.-m_{4}\left(v_{1} v_{3}+v_{2} v_{4}-\sigma v_{6}\right)\right]+\alpha\left(k_{3} w_{5}-l_{3} u_{5}-m_{3} v_{5}\right)\right\}
\end{aligned}\right.
$$

then driven systems (5) and (6) will achieve combination synchronization with response system (7).

Proof. Construct the following Lyapunov function:

$$
V=\frac{1}{2}\left(e_{1}^{2}+e_{2}^{2}+e_{3}^{2}+e_{4}^{2}+e_{5}^{2}+e_{6}^{2}\right) .
$$

Taking the time derivative of $V$ along the trajectory of error dynamical system (11) yields:

$$
\begin{aligned}
\dot{V}= & e_{1} \dot{e}_{1}+e_{2} \dot{e}_{2}+e_{3} \dot{e}_{3}+e_{4} \dot{e}_{4}+e_{5} \dot{e}_{5}+e_{6} \dot{e}_{6} \\
= & e_{1}\left\{k_{1}\left[\alpha\left(w_{3}-w_{1}\right)+w_{6}\right]-l_{1}\left[\alpha\left(u_{3}-u_{1}\right)+u_{6}\right]-m_{1}\left[\alpha\left(v_{3}-v_{1}\right)+v_{6}\right]+k_{1} U_{1}\right\} \\
& +e_{2}\left[k_{1} \alpha\left(w_{4}-w_{2}\right)-l_{1} \alpha\left(u_{4}-u_{2}\right)-m_{1} \alpha\left(v_{4}-v_{2}\right)+k_{1} U_{2}\right] \\
& +e_{3}\left[k_{2}\left(\gamma w_{1}-w_{3}-w_{1} w_{5}\right)-l_{2}\left(\gamma u_{1}-u_{3}-u_{1} u_{5}\right)-m_{2}\left(\gamma v_{1}-v_{3}-v_{1} v_{5}\right)+k_{2} U_{3}\right] \\
& +e_{4}\left[k_{2}\left(\gamma w_{2}-w_{4}-w_{2} w_{5}\right)-l_{2}\left(\gamma u_{2}-u_{4}-u_{2} u_{5}\right)-m_{2}\left(\gamma v_{2}-v_{4}-v_{2} v_{5}\right)+k_{2} U_{4}\right] \\
& +e_{5}\left[k_{3}\left(w_{1} w_{3}+w_{2} w_{4}-\beta w_{5}+w_{6}\right)-l_{3}\left(u_{1} u_{3}+u_{2} u_{4}-\beta u_{5}+u_{6}\right)-m_{3}\left(v_{1} v_{3}+v_{2} v_{4}-\beta v_{5}+v_{6}\right)+k_{3} U_{5}\right] \\
& +e_{6}\left[k_{4}\left(w_{1} w_{3}+w_{2} w_{4}-\sigma w_{6}\right)-l_{4}\left(u_{1} u_{3}+u_{2} u_{4}-\sigma u_{6}\right)-m_{4}\left(v_{1} v_{3}+v_{2} v_{4}-\sigma v_{6}\right)+k_{4} U_{6}\right] .
\end{aligned}
$$


Substituting Equation (12) into Equation (14), then:

$$
\begin{aligned}
& \dot{V}=e_{1}\left\{k_{1}\left[\alpha\left(w_{3}-w_{1}\right)+w_{6}\right]-l_{1}\left[\alpha\left(u_{3}-u_{1}\right)+u_{6}\right]-m_{1}\left[\alpha\left(v_{3}-v_{1}\right)+v_{6}\right]-\left[k_{1} w_{1}-l_{1} u_{1}-m_{1} v_{1}\right.\right. \\
& \left.\left.+k_{1} \alpha\left(w_{3}-w_{1}\right)+k_{1} w_{6}-l_{1} \alpha\left(u_{3}-u_{1}\right)-l_{1} u_{6}-m_{1} \alpha\left(v_{3}-v_{1}\right)-m_{1} v_{6}-\alpha\left(k_{1} w_{2}-l_{1} u_{2}-m_{1} v_{2}\right)\right]\right\} \\
& +e_{2}\left\{k_{1} \alpha\left(w_{4}-w_{2}\right)-l_{1} \alpha\left(u_{4}-u_{2}\right)-m_{1} \alpha\left(v_{4}-v_{2}\right)-\left[k_{1} w_{2}-l_{1} u_{2}-m_{1} v_{2}\right.\right. \\
& \left.\left.+k_{1} \alpha\left(w_{4}-w_{2}\right)-l_{1} \alpha\left(u_{4}-u_{2}\right)-m_{1} \alpha\left(v_{4}-v_{2}\right)+\alpha\left(k_{1} w_{1}-l_{1} u_{1}-m_{1} v_{1}\right)-\gamma\left(k_{2} w_{3}-l_{2} u_{3}-m_{2} v_{3}\right)\right]\right\} \\
& +e_{3}\left\{k_{2}\left(\gamma w_{1}-w_{3}-w_{1} w_{5}\right)-l_{2}\left(\gamma u_{1}-u_{3}-u_{1} u_{5}\right)-m_{2}\left(\gamma v_{1}-v_{3}-v_{1} v_{5}\right)-\left[k_{2} w_{3}-l_{2} u_{3}-m_{2} v_{3}\right.\right. \\
& +k_{2}\left(\gamma w_{1}-w_{3}-w_{1} w_{5}\right)-l_{2}\left(\gamma u_{1}-u_{3}-u_{1} u_{5}\right)-m_{2}\left(\gamma v_{1}-v_{3}-v_{1} v_{5}\right) \\
& \left.\left.+\gamma\left(k_{1} w_{2}-l_{1} u_{2}-m_{1} v_{2}\right)-\beta\left(k_{2} w_{4}-l_{2} u_{4}-m_{2} v_{4}\right)\right]\right\} \\
& +e_{4}\left\{k_{2}\left(\gamma w_{2}-w_{4}-w_{2} w_{5}\right)-l_{2}\left(\gamma u_{2}-u_{4}-u_{2} u_{5}\right)-m_{2}\left(\gamma v_{2}-v_{4}-v_{2} v_{5}\right)-\left[k_{2} w_{4}-l_{2} u_{4}-m_{2} v_{4}\right.\right. \\
& +k_{2}\left(\gamma w_{2}-w_{4}-w_{2} w_{5}\right)-l_{2}\left(\gamma u_{2}-u_{4}-u_{2} u_{5}\right)-m_{2}\left(\gamma v_{2}-v_{4}-v_{2} v_{5}\right) \\
& \left.\left.+\beta\left(k_{2} w_{3}-l_{2} u_{3}-m_{2} v_{3}\right)-\sigma\left(k_{3} w_{5}-l_{3} u_{5}-m_{3} v_{5}\right)\right]\right\}+e_{5}\left\{k_{3}\left(w_{1} w_{3}+w_{2} w_{4}-\beta w_{5}+w_{6}\right)\right. \\
& -l_{3}\left(u_{1} u_{3}+u_{2} u_{4}-\beta u_{5}+u_{6}\right)-m_{3}\left(v_{1} v_{3}+v_{2} v_{4}-\beta v_{5}+v_{6}\right)-\left[k_{3} w_{5}-l_{3} u_{5}-m_{3} v_{5}\right. \\
& +k_{3}\left(w_{1} w_{3}+w_{2} w_{4}-\beta w_{5}+w_{6}\right)-l_{3}\left(u_{1} u_{3}+u_{2} u_{4}-\beta u_{5}+u_{6}\right)-m_{3}\left(v_{1} v_{3}+v_{2} v_{4}-\beta v_{5}+v_{6}\right) \\
& \left.\left.+\sigma\left(k_{2} w_{4}-l_{2} u_{4}-m_{2} v_{4}\right)-\alpha\left(k_{4} w_{6}-l_{4} u_{6}-m_{4} v_{6}\right)\right]\right\}+e_{6}\left\{k_{4}\left(w_{1} w_{3}+w_{2} w_{4}-\sigma w_{6}\right)\right. \\
& -l_{4}\left(u_{1} u_{3}+u_{2} u_{4}-\sigma u_{6}\right)-m_{4}\left(v_{1} v_{3}+v_{2} v_{4}-\sigma v_{6}\right)-\left[k_{4} w_{6}-l_{4} u_{6}-m_{4} v_{6}+k_{4}\left(w_{1} w_{3}+w_{2} w_{4}-\sigma w_{6}\right)\right. \\
& \left.\left.-l_{4}\left(u_{1} u_{3}+u_{2} u_{4}-\sigma u_{6}\right)-m_{4}\left(v_{1} v_{3}+v_{2} v_{4}-\sigma v_{6}\right)+\alpha\left(k_{3} w_{5}-l_{3} u_{5}-m_{3} v_{5}\right)\right]\right\} \\
& =e_{1}\left(-e_{1}+\alpha e_{2}\right)+e_{2}\left(-e_{2}-\alpha e_{1}+\gamma e_{3}\right)+e_{3}\left(-e_{3}-\gamma e_{2}+\beta e_{4}\right)+e_{4}\left(-e_{4}-\beta e_{3}+\sigma e_{5}\right) \\
& +e_{5}\left(-e_{5}-\sigma e_{4}+\alpha e_{6}\right)+e_{6}\left(-e_{6}-\alpha e_{5}\right) \\
& =-e_{1}^{2}-e_{2}^{2}-e_{3}^{2}-e_{4}^{2}-e_{5}^{2}-e_{6}^{2} \text {. }
\end{aligned}
$$

Since $\dot{V} \leq 0$ as $t \rightarrow \infty$, according to the Lyapunov stability theory, we know $e_{i} \rightarrow 0(i=1,2,3,4,5,6)$, i.e., $\lim _{t \rightarrow \infty}\|e\|=0$. Therefore, drive systems (5) and (6) will achieve combination synchronization with the response system (7).

This completes the proof.

The following corollaries can be easily obtained from Theorem 1.

Corollary 1. (i) Suppose that $l_{1}=l_{2}=l_{3}=l_{4}=0$ and $k_{1}=k_{2}=k_{3}=k_{4}=1$, and if the controllers are chosen as follows:

$$
\left\{\begin{aligned}
U_{1}= & -\left\{\left(w_{1}-m_{1} v_{1}\right)+\left[\alpha\left(w_{3}-w_{1}\right)+w_{6}-m_{1} \alpha\left(v_{3}-v_{1}\right)-m_{1} v_{6}\right]-\alpha\left(w_{2}-m_{1} v_{2}\right)\right\} \\
U_{2}= & \left.-\left\{\left(w_{2}-m_{1} v_{2}\right)+\left[\alpha\left(w_{4}-w_{2}\right)-m_{1} \alpha\left(v_{4}-v_{2}\right)\right]+\alpha\left(w_{1}-m_{1} v_{1}\right)-\gamma\left(w_{3}-m_{2} v_{3}\right)\right\}\right\} \\
U_{3}= & -\left\{\left(w_{3}-m_{2} v_{3}\right)+\left[\left(\gamma w_{1}-w_{3}-w_{1} w_{5}\right)-m_{2}\left(\gamma v_{1}-v_{3}-v_{1} v_{5}\right)\right]+\gamma\left(w_{2}-m_{1} v_{2}\right)-\beta\left(w_{4}-m_{2} v_{4}\right)\right\} \\
U_{4}= & -\left\{\left(w_{4}-m_{2} v_{4}\right)+\left[\left(\gamma w_{2}-w_{4}-w_{2} w_{5}\right)-m_{2}\left(\gamma v_{2}-v_{4}-v_{2} v_{5}\right)\right]+\beta\left(w_{3}-m_{2} v_{3}\right)-\sigma\left(w_{5}-m_{3} v_{5}\right)\right\} \\
U_{5}= & -\left\{\left(w_{5}-m_{3} v_{5}\right)+\left[\left(w_{1} w_{3}+w_{2} w_{4}-\beta w_{5}+w_{6}\right)-m_{3}\left(v_{1} v_{3}+v_{2} v_{4}-\beta v_{5}+v_{6}\right)\right]\right. \\
& \left.+\sigma\left(w_{4}-m_{2} v_{4}\right)-\alpha\left(w_{6}-m_{4} v_{6}\right)\right\} \\
U_{6}= & -\left\{\left(w_{6}-m_{4} v_{6}\right)+\left[\left(w_{1} w_{3}+w_{2} w_{4}-\sigma w_{6}\right)-m_{4}\left(v_{1} v_{3}+v_{2} v_{4}-\sigma v_{6}\right)\right]+\alpha\left(w_{5}-m_{3} v_{5}\right)\right\}
\end{aligned}\right.
$$

then drive system (6) will achieve projective synchronization with response system (7). 
(ii) Suppose that $m_{1}=m_{2}=m_{3}=m_{4}=0$ and $k_{1}=k_{2}=k_{3}=k_{4}=1$, and if the controllers are chosen as follows:

$$
\left\{\begin{aligned}
U_{1}= & -\left\{\left(w_{1}-l_{1} u_{1}\right)+\left[\alpha\left(w_{3}-w_{1}\right)+w_{6}-l_{1} \alpha\left(u_{3}-u_{1}\right)-l_{1} u_{6}\right]-\alpha\left(w_{2}-l_{1} u_{2}\right)\right\} \\
U_{2}= & -\left\{\left(w_{2}-l_{1} u_{2}\right)+\left[\alpha\left(w_{4}-w_{2}\right)-l_{1} \alpha\left(u_{4}-u_{2}\right)\right]+\alpha\left(w_{1}-l_{1} u_{1}\right)-\gamma\left(w_{3}-l_{2} u_{3}\right)\right\} \\
U_{3}= & -\left\{\left(w_{3}-l_{2} u_{3}\right)+\left[\left(\gamma w_{1}-w_{3}-w_{1} w_{5}\right)-l_{2}\left(\gamma u_{1}-u_{3}-u_{1} u_{5}\right)\right]+\gamma\left(w_{2}-l_{1} u_{2}\right)-\beta\left(w_{4}-l_{2} u_{4}\right)\right\} \\
U_{4}= & -\left\{\left(w_{4}-l_{2} u_{4}\right)+\left[\left(\gamma w_{2}-w_{4}-w_{2} w_{5}\right)-l_{2}\left(\gamma u_{2}-u_{4}-u_{2} u_{5}\right)\right]+\beta\left(w_{3}-l_{2} u_{3}\right)-\sigma\left(w_{5}-l_{3} u_{5}\right)\right\} \\
U_{5}= & -\left\{\left(w_{5}-l_{3} u_{5}\right)+\left[\left(w_{1} w_{3}+w_{2} w_{4}-\beta w_{5}+w_{6}\right)-l_{3}\left(u_{1} u_{3}+u_{2} u_{4}-\beta u_{5}+u_{6}\right)\right]\right. \\
& \left.+\sigma\left(w_{4}-l_{2} u_{4}\right)-\alpha\left(w_{6}-l_{4} u_{6}\right)\right\} \\
U_{6}= & -\left\{\left(w_{6}-l_{4} u_{6}\right)+\left[\left(w_{1} w_{3}+w_{2} w_{4}-\sigma w_{6}\right)-l_{4}\left(u_{1} u_{3}+u_{2} u_{4}-\sigma u_{6}\right)\right]+\alpha\left(w_{5}-l_{3} u_{5}\right)\right\}
\end{aligned}\right.
$$

then drive system (5) will achieve projective synchronization with response system (7).

Corollary 2. Suppose that $l_{1}=l_{2}=l_{3}=l_{4}=0, m_{1}=m_{2}=m_{3}=m_{4}=0$ and $k_{1}=k_{2}=k_{3}=$ $k_{4}=1$, and if the controllers are chosen as follows:

$$
\left\{\begin{array}{l}
U_{1}=-\left[w_{1}+\alpha\left(w_{3}-w_{1}\right)+w_{6}-\alpha w_{2}\right] \\
U_{2}=-\left[w_{2}+\alpha\left(w_{4}-w_{2}\right)+\alpha w_{1}-\gamma w_{3}\right] \\
U_{3}=-\left(w_{3}+\gamma w_{1}-w_{3}-w_{1} w_{5}+\gamma w_{2}-\beta w_{4}\right) \\
U_{4}=-\left(w_{4}+\gamma w_{2}-w_{4}-w_{2} w_{5}+\beta w_{3}-\sigma w_{5}\right) \\
U_{5}=-\left(w_{5}+w_{1} w_{3}+w_{2} w_{4}-\beta w_{5}+w_{6}+\sigma w_{4}-\alpha w_{6}\right) \\
U_{6}=-\left(w_{6}+w_{1} w_{3}+w_{2} w_{4}-\sigma w_{6}+\alpha w_{5}\right)
\end{array}\right.
$$

then system (7) is stabilized to the equilibrium, $O(0,0,0,0,0,0)$.

Remark 1: The proofs of Corollary 1 and Corollary 2 are similar to those of theorem 1, so we omitted them.

In the following, numerical experiments are given to demonstrate our results. The fourth-order Runge-Kutta method is used with a time step size of 0.001 . The system parameters are given as $\alpha=8, \beta=5, \gamma=50$ and $\sigma=15$, so that the complex Lorenz system exhibits hyperchaotic behavior. We assume $k_{1}=k_{2}=k_{3}=k_{4}=1, l_{1}=l_{2}=l_{3}=l_{4}=1$ and $m_{1}=m_{2}=m_{3}=m_{4}=1$, and the initial states for drive systems (5) and (6) and response system (7) are arbitrarily given by $\left(x_{11}(0), x_{21}(0), x_{31}(0), x_{41}(0)\right)=(4-0.3 i, 2.2-0.8 i, 4.9,1.1),\left(x_{12}(0), x_{22}(0), x_{32}(0), x_{42}(0)\right)=$ $(4.4-0.6 i, 3.3-1.4 i, 5.3,1.4)$ and $\left(x_{13}(0), x_{23}(0), x_{33}(0), x_{43}(0)\right)=(4.6-1.8 i, 1.6-$ $1.9 i, 2.5,2)$, i.e., $\quad\left(u_{1}(0), u_{2}(0), u_{3}(0), u_{4}(0), u_{5}(0), u_{6}(0)\right) \quad=\quad(4,-0.3,2.2,-0.8,4.9,1.1)$, $\left(v_{1}(0), v_{2}(0), v_{3}(0), v_{4}(0), v_{5}(0), v_{6}(0)\right) \quad=\quad(4.4,-0.6,3.3,-1.4,5.3,1.4) \quad$ and $\left(w_{1}(0), w_{2}(0), w_{3}(0), w_{4}(0), w_{5}(0), w_{6}(0)\right)=(4.6,-1.8,1.6,-1.9,2.5,2), \quad$ respectively. $\quad$ The corresponding numerical results are shown in Figures 1 and 2. Figure 1 displays the time response of the combination synchronization errors, $e_{1}, e_{2}, e_{3}, e_{4}, e_{5} a d_{d}$. The errors converge to zero, which implies that systems (5), (6) and (7) have achieved combination synchronization. Figures 2 depicts the time responses of the states, $u_{1}+v_{1}$ versus $w_{1}, u_{2}+v_{2}$ versus $w_{2}, u_{3}+v_{3}$ versus $w_{3}, u_{4}+v_{4}$ versus $w_{4}, u_{5}+v_{5}$ versus $w_{5}$ and $u_{6}+v_{6}$ versus $w_{6}$, respectively. Next, suppose that $k_{1}=k_{2}=k_{3}=k_{4}=1$, $l_{1}=l_{2}=l_{3}=l_{4}=0$ and $m_{1}=m_{2}=m_{3}=m_{4}=0$. The time evolution of the states, 
$w_{1}, w_{2}, w_{3}, w_{4}, w_{5}, w_{6}$, of system (7) with controller (18) are displayed in Figure 3, which illustrates that system (7) is stabilized to the equilibrium, $O(0,0,0,0,0,0)$.

Figure 1. Combination synchronization errors, $e_{1}, e_{2}, e_{3}, e_{4}, e_{5}$ and $e_{6}$, between drive systems (5) and (6) and response system (7).
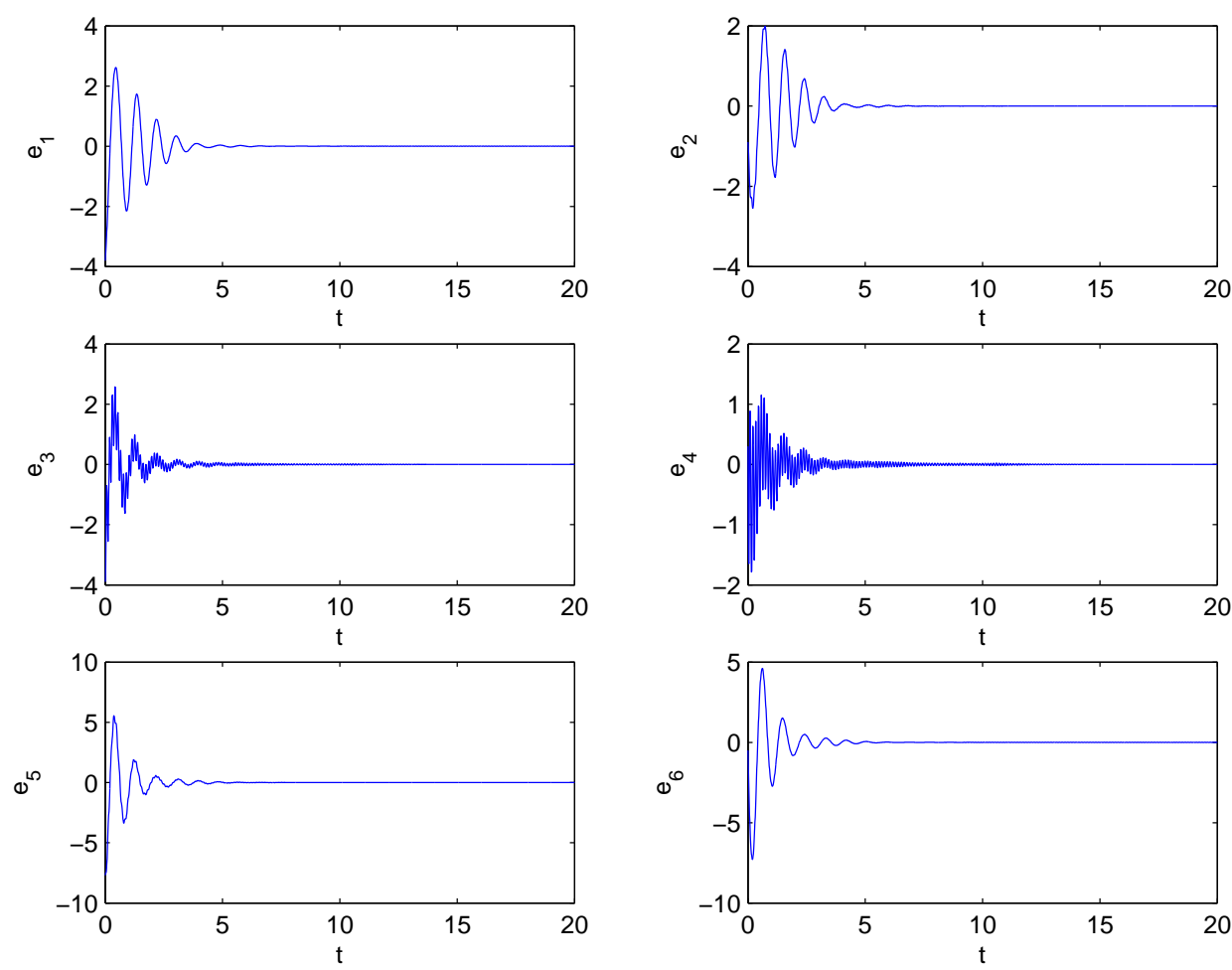

\section{Combination Synchronization among Different Nonlinear Complex Hyperchaotic Systems}

In this section, we investigate the combination synchronization among three different nonlinear complex hyperchaotic systems. The hyperchaotic complex Lorenz system [6] and the hyperchaotic complex Chen system [5], respectively, describe the drive systems:

$$
\left\{\begin{array}{l}
\dot{x}_{1}=\alpha_{1}\left(x_{2}-x_{1}\right)+x_{4}, \\
\dot{x}_{2}=\gamma_{1} x_{1}-x_{2}-x_{1} x_{3}, \\
\dot{x}_{3}=\frac{1}{2}\left(\bar{x}_{1} x_{2}+x_{1} \bar{x}_{2}\right)-\beta_{1} x_{3}+x_{4}, \\
\dot{x}_{4}=\frac{1}{2}\left(\bar{x}_{1} x_{2}+x_{1} \bar{x}_{2}\right)-\sigma_{1} x_{4},
\end{array}\right.
$$

and

$$
\left\{\begin{array}{l}
\dot{y}_{1}=\alpha_{2}\left(y_{2}-y_{1}\right), \\
\dot{y}_{2}=\left(\gamma_{2}-\alpha_{2}\right) y_{1}-y_{1} y_{3}+\gamma_{2} y_{2}+y_{4}, \\
\dot{y}_{3}=\frac{1}{2}\left(\bar{y}_{1} y_{2}+y_{1} \bar{y}_{2}\right)-\beta_{2} y_{3}+y_{4}, \\
\dot{y}_{4}=\frac{1}{2}\left(\bar{y}_{1} y_{2}+y_{1} \bar{y}_{2}\right)-d_{2} y_{4},
\end{array}\right.
$$


Figure 2. Time responses for states $u_{i}+v_{i}$ versus $w_{i}, i=1,2, \ldots, 6$, respectively.
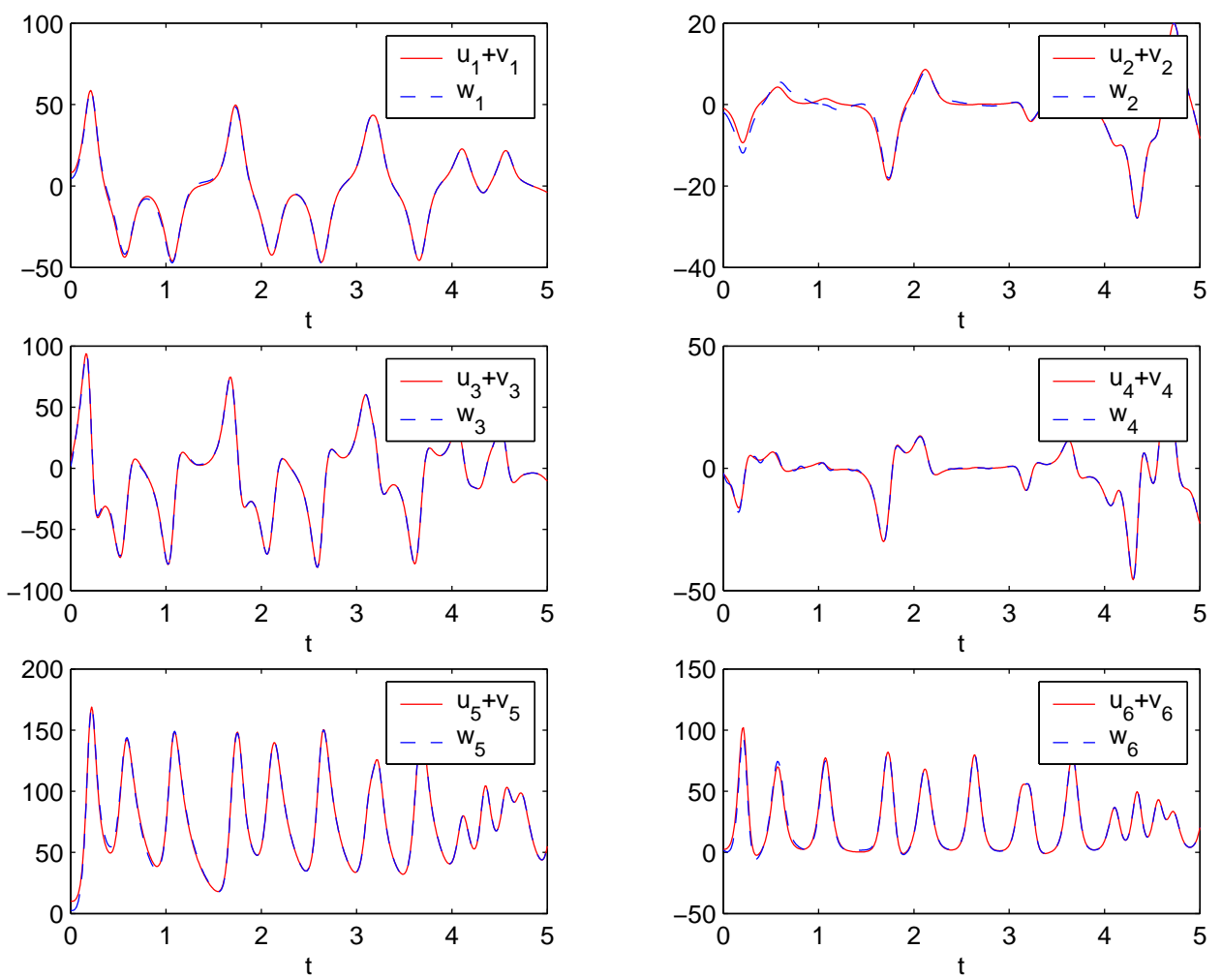

Figure 3. Time evolution of the states for system (7).
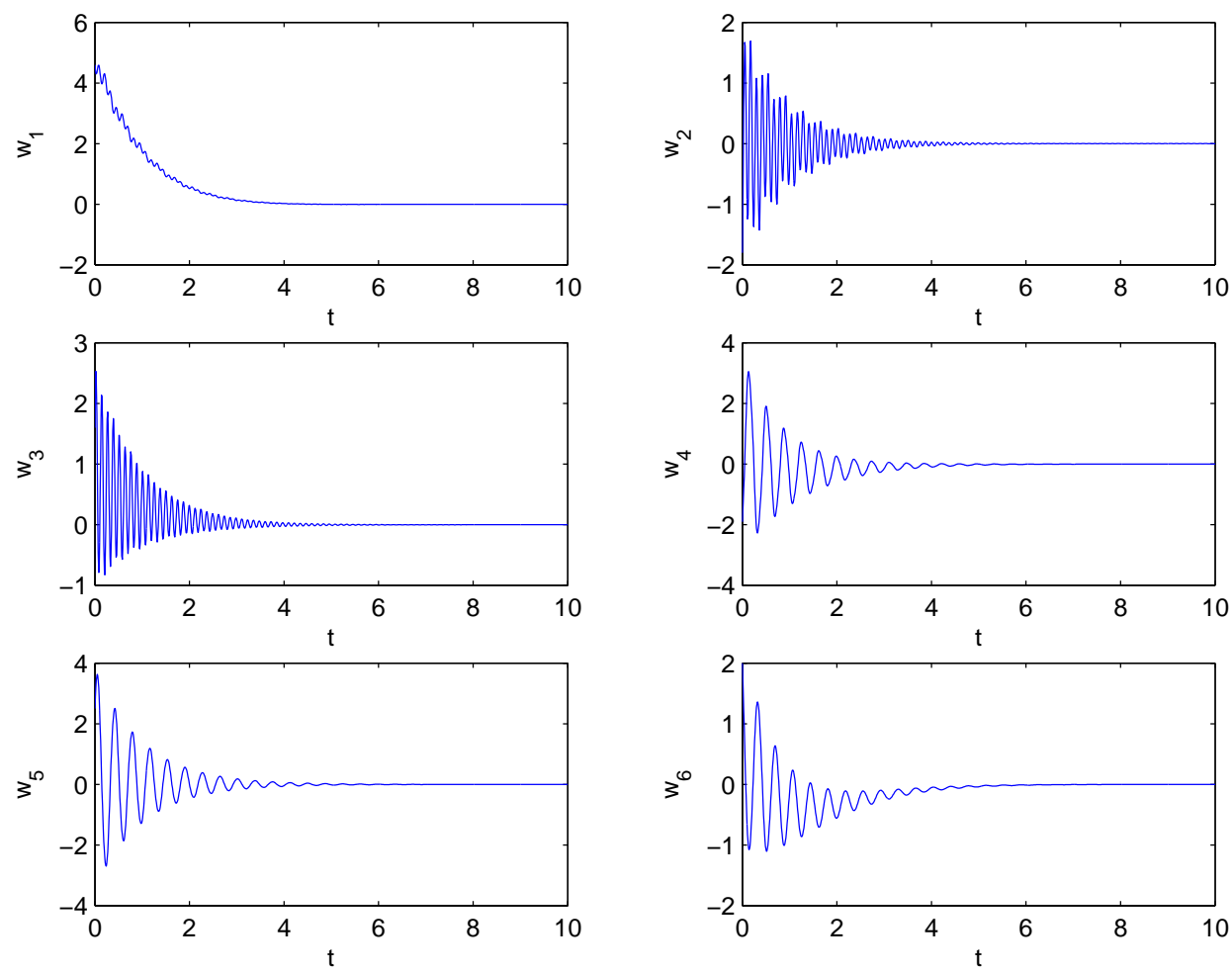
and the hyperchaotic complex Lü system system [7] is the response system given by:

$$
\left\{\begin{array}{l}
\dot{z}_{1}=\rho_{3}\left(z_{2}-z_{1}\right)+z_{4}+U_{1}+i U_{2} \\
\dot{z}_{2}=\nu_{3} z_{2}-z_{1} z_{3}+z_{4}+U_{3}+i U_{4} \\
\dot{z}_{3}=\frac{1}{2}\left(\bar{z}_{1} z_{2}+z_{1} \bar{z}_{2}\right)-\mu_{3} z_{3}+U_{5} \\
\dot{z}_{4}=\frac{1}{2}\left(\bar{z}_{1} z_{2}+z_{1} \bar{z}_{2}\right)-\sigma_{3} z_{4}+U_{6}
\end{array}\right.
$$

where $\alpha_{1}, \beta_{1}, \gamma_{1}, \sigma_{1}, \alpha_{2}, \beta_{2}, \gamma_{2}, d_{2}, \rho_{3}, \nu_{3}, \mu_{3}$ and $\sigma_{3}$ are positive parameters, $x_{1}=u_{1}+i u_{2}, x_{2}=$ $u_{3}+i u_{4}, y_{1}=v_{1}+i v_{2}, y_{2}=v_{3}+i v_{4}, z_{1}=w_{1}+i w_{2}, z_{2}=w_{3}+i w_{4}$ are complex variables and $i=\sqrt{-1} ; u_{i}, v_{i}, w_{i}(i=1,2,3,4), x_{3}=u_{5}, x_{4}=u_{6}, y_{3}=v_{5}, y_{4}=v_{6}, z_{3}=w_{5}$ and $z_{4}=w_{6}$ are real variables. The overbar represents the complex conjugate function. $U_{1}, U_{2}, U_{3}, U_{4}, U_{5}$ and $U_{6}$ are real control functions to be determined.

For the convenience of the following discussions, we assume $A=\operatorname{diag}\left(l_{1}, l_{2}, l_{3}, l_{4}\right), B=$ $\operatorname{diag}\left(m_{1}, m_{2}, m_{3}, m_{4}\right)$ and $C=\operatorname{diag}\left(k_{1}, k_{2}, k_{3}, k_{4}\right)$ in our synchronization scheme.

We define error states between drive systems (19)-(20) and response system (21) as:

$$
\left\{\begin{aligned}
e_{1}+i e_{2} & =k_{1} z_{1}-l_{1} x_{1}-m_{1} y_{1} \\
e_{3}+i e_{4} & =k_{2} z_{2}-l_{2} x_{2}-m_{2} y_{2} \\
e_{4} & =k_{3} z_{3}-l_{3} x_{3}-m_{3} y_{3} \\
e_{5} & =k_{4} z_{4}-l_{4} x_{4}-m_{4} y_{4}
\end{aligned}\right.
$$

such that:

$$
\left\{\begin{array}{l}
\lim _{t \rightarrow \infty}\left\|k_{1} z_{1}-l_{1} x_{1}-m_{1} y_{1}\right\|=0 \\
\lim _{t \rightarrow \infty}\left\|k_{2} z_{2}-l_{2} x_{2}-m_{2} y_{2}\right\|=0 \\
\lim _{t \rightarrow \infty}\left\|k_{3} z_{3}-l_{3} x_{3}-m_{3} y_{3}\right\|=0 \\
\lim _{t \rightarrow \infty}\left\|k_{4} z_{4}-l_{4} x_{4}-m_{4} y_{4}\right\|=0
\end{array}\right.
$$

Separating the real and imagery parts of Equation (22) gets the following:

$$
\left\{\begin{array}{l}
e_{1}=\left(k_{1} w_{1}-l_{1} u_{1}-m_{1} v_{1}\right), \\
e_{2}=\left(k_{1} w_{2}-l_{1} u_{2}-m_{1} v_{2}\right), \\
e_{3}=\left(k_{2} w_{3}-l_{2} u_{3}-m_{2} v_{3}\right), \\
e_{4}=\left(k_{2} w_{4}-l_{2} u_{4}-m_{2} v_{4}\right), \\
e_{5}=\left(k_{3} w_{5}-l_{3} u_{5}-m_{3} v_{5}\right), \\
e_{6}=\left(k_{4} w_{6}-l_{4} u_{6}-m_{4} v_{6}\right) .
\end{array}\right.
$$


Thus, we have the following error dynamical system:

$$
\left\{\begin{array}{l}
\dot{e}_{1}=k_{1}\left[\rho_{3}\left(w_{3}-w_{1}\right)+w_{6}\right]-l_{1}\left[\alpha_{1}\left(u_{3}-u_{1}\right)+u_{6}\right]-m_{1} \alpha_{2}\left(v_{3}-v_{1}\right)+k_{1} U_{1} \\
\dot{e}_{2}=k_{1} \rho_{3}\left(w_{4}-w_{2}\right)-l_{1} \alpha_{1}\left(u_{4}-u_{2}\right)-m_{1} \alpha_{2}\left(v_{4}-v_{2}\right)+k_{1} U_{2} \\
\dot{e}_{3}=k_{2}\left(-w_{1} w_{5}+\nu_{3} w_{3}+w_{6}\right)-l_{2}\left(\gamma_{1} u_{1}-u_{3}-u_{1} u_{5}\right)-m_{2}\left[\left(\gamma_{2}-\alpha_{2}\right) v_{1}-v_{1} v_{5}+\gamma_{2} v_{3}+v_{6}\right]+k_{2} U_{3} \\
\dot{e}_{4}=k_{2}\left(-w_{2} w_{5}+\nu_{3} w_{4}\right)-l_{2}\left(\gamma_{1} u_{2}-u_{4}-u_{2} u_{5}\right)-m_{2}\left[\left(\gamma_{2}-\alpha_{2}\right) v_{2}-v_{2} v_{5}+\gamma_{2} v_{4}\right]+k_{2} U_{4} \\
\dot{e}_{5}=k_{3}\left(w_{1} w_{3}+w_{2} w_{4}-\mu_{3} w_{5}\right)-l_{3}\left(u_{1} u_{3}+u_{2} u_{4}-\beta_{1} u_{5}+u_{6}\right)-m_{3}\left(v_{1} v_{3}+v_{2} v_{4}-\beta_{2} v_{5}+v_{6}\right)+k_{3} U_{5} \\
\dot{e}_{6}=k_{4}\left(w_{1} w_{3}+w_{2} w_{4}-\sigma_{3} w_{6}\right)-l_{4}\left(u_{1} u_{3}+u_{2} u_{4}-\sigma_{1} u_{6}\right)-m_{4}\left(v_{1} v_{3}+v_{2} v_{4}-d_{2} v_{6}\right)+k_{4} U_{6}
\end{array}\right.
$$

Similar to Section 3, we have the following results.

Theorem 2. If the controllers are chosen as:

$$
\left\{\begin{aligned}
U_{1}= & -\frac{1}{k_{1}}\left\{\left(k_{1} w_{1}-l_{1} u_{1}-m_{1} v_{1}\right)+\left[k_{1} \rho_{3}\left(w_{3}-w_{1}\right)+k_{1} w_{6}-l_{1} \alpha_{1}\left(u_{3}-u_{1}\right)-l_{1} u_{6}-m_{1} \alpha_{2}\left(v_{3}-v_{1}\right)\right]\right. \\
& \left.-\alpha_{1}\left(k_{1} w_{2}-l_{1} u_{2}-m_{1} v_{2}\right)\right\} \\
U_{2}= & -\frac{1}{k_{1}}\left\{\left(k_{1} w_{2}-l_{1} u_{2}-m_{1} v_{2}\right)+\left[k_{1} \rho_{3}\left(w_{4}-w_{2}\right)-l_{1} \alpha_{1}\left(u_{4}-u_{2}\right)-m_{1} \alpha_{2}\left(v_{4}-v_{2}\right)\right]\right. \\
& \left.+\alpha_{1}\left(k_{1} w_{1}-l_{1} u_{1}-m_{1} v_{1}\right)-\alpha_{2}\left(k_{2} w_{3}-l_{2} u_{3}-m_{2} v_{3}\right)\right\}, \\
U_{3}= & -\frac{1}{k_{2}}\left\{\left(k_{2} w_{3}-l_{2} u_{3}-m_{2} v_{3}\right)+\left[k_{2}\left(-w_{1} w_{5}+\nu_{3} w_{3}+w_{6}\right)-l_{2}\left(\gamma_{1} u_{1}-u_{3}-u_{1} u_{5}\right)-m_{2}\left(\gamma_{2}-\alpha_{2}\right) v_{1}\right.\right. \\
& \left.\left.+m_{2} v_{1} v_{5}-m_{2} \gamma_{2} v_{3}-m_{2} v_{6}\right]+\alpha_{2}\left(k_{1} w_{2}-l_{1} u_{2}-m_{1} v_{2}\right)-\beta_{1}\left(k_{2} w_{4}-l_{2} u_{4}-m_{2} v_{4}\right)\right\}, \\
U_{4}= & -\frac{1}{k_{2}}\left\{\left(k_{2} w_{4}-l_{2} u_{4}-m_{2} v_{4}\right)+\left[k_{2}\left(-w_{2} w_{5}+\nu_{3} w_{4}\right)-l_{2}\left(\gamma_{1} u_{2}-u_{4}-u_{2} u_{5}\right)-m_{2}\left(\gamma_{2}-\alpha_{2}\right) v_{2}\right.\right. \\
& \left.\left.+m_{2} v_{2} v_{5}-m_{2} \gamma_{2} v_{4}\right]+\beta_{1}\left(k_{2} w_{3}-l_{2} u_{3}-m_{2} v_{3}\right)-\beta_{2}\left(k_{3} w_{5}-l_{3} u_{5}-m_{3} v_{5}\right)\right\}, \\
U_{5}= & -\frac{1}{k_{3}}\left\{\left(k_{3} w_{5}-l_{3} u_{5}-m_{3} v_{5}\right)+\left[k_{3}\left(w_{1} w_{3}+w_{2} w_{4}-\mu_{3} w_{5}\right)-l_{3}\left(u_{1} u_{3}+u_{2} u_{4}-\beta_{1} u_{5}+u_{6}\right)\right.\right. \\
& \left.\left.-m_{3}\left(v_{1} v_{3}+v_{2} v_{4}-\beta_{2} v_{5}+v_{6}\right)\right]+\beta_{2}\left(k_{2} w_{4}-l_{2} u_{4}-m_{2} v_{4}\right)-\gamma_{1}\left(k_{4} w_{6}-l_{4} u_{6}-m_{4} v_{6}\right)\right\}, \\
U_{6}= & -\frac{1}{k_{4}}\left\{\left(k_{4} w_{6}-l_{4} u_{6}-m_{4} v_{6}\right)+\left[k_{4}\left(w_{1} w_{3}+w_{2} w_{4}-\sigma_{3} w_{6}\right)-l_{4}\left(u_{1} u_{3}+u_{2} u_{4}-\sigma_{1} u_{6}\right)\right.\right. \\
& \left.\left.-m_{4}\left(v_{1} v_{3}+v_{2} v_{4}-d_{2} v_{6}\right)\right]+\gamma_{1}\left(k_{3} w_{5}-l_{3} u_{5}-m_{3} v_{5}\right)\right\},
\end{aligned}\right.
$$

then drive systems (19) and (20) will achieve combination synchronization with response system (21). 
Corollary 3. (i) Suppose that $l_{1}=l_{2}=l_{3}=l_{4}=0$ and $k_{1}=k_{2}=k_{3}=k_{4}=1$, and if the controllers are chosen as follows:

$$
\left\{\begin{aligned}
U_{1}= & -\left\{\left(w_{1}-m_{1} v_{1}\right)+\left[\rho_{3}\left(w_{3}-w_{1}\right)+w_{6}-m_{1} \alpha_{2}\left(v_{3}-v_{1}\right)\right]-\alpha_{1}\left(w_{2}-m_{1} v_{2}\right)\right\} \\
U_{2}= & -\left\{\left(w_{2}-m_{1} v_{2}\right)+\left[\rho_{3}\left(w_{4}-w_{2}\right)-m_{1} \alpha_{2}\left(v_{4}-v_{2}\right)\right]+\alpha_{1}\left(w_{1}-m_{1} v_{1}\right)-\alpha_{2}\left(w_{3}-m_{2} v_{3}\right)\right\} \\
U_{3}= & -\left\{\left(w_{3}-m_{2} v_{3}\right)+\left[\left(-w_{1} w_{5}+\nu_{3} w_{3}+w_{6}\right)-m_{2}\left(\gamma_{2}-\alpha_{2}\right) v_{1}+m_{2} v_{1} v_{5}-m_{2} \gamma_{2} v_{3}-m_{2} v_{6}\right]\right. \\
& \left.+\alpha_{2}\left(w_{2}-m_{1} v_{2}\right)-\beta_{1}\left(w_{4}-m_{2} v_{4}\right)\right\} \\
U_{4}= & -\left\{\left(w_{4}-m_{2} v_{4}\right)+\left[\left(-w_{2} w_{5}+\nu_{3} w_{4}\right)-m_{2}\left(\gamma_{2}-\alpha_{2}\right) v_{2}+m_{2} v_{2} v_{5}-m_{2} \gamma_{2} v_{4}\right]\right. \\
& \left.+\beta_{1}\left(w_{3}-m_{2} v_{3}\right)-\beta_{2}\left(w_{5}-m_{3} v_{5}\right)\right\} \\
U_{5}= & -\left\{\left(w_{5}-m_{3} v_{5}\right)+\left[\left(w_{1} w_{3}+w_{2} w_{4}-\mu_{3} w_{5}\right)-m_{3}\left(v_{1} v_{3}+v_{2} v_{4}-\beta_{2} v_{5}+v_{6}\right)\right]\right. \\
& \left.+\beta_{2}\left(w_{4}-m_{2} v_{4}\right)-\gamma_{1}\left(w_{6}-m_{4} v_{6}\right)\right\} \\
U_{6}= & -\left\{\left(w_{6}-m_{4} v_{6}\right)+\left[\left(w_{1} w_{3}+w_{2} w_{4}-\sigma_{3} w_{6}\right)-m_{4}\left(v_{1} v_{3}+v_{2} v_{4}-d_{2} v_{6}\right)\right]+\gamma_{1}\left(w_{5}-m_{3} v_{5}\right)\right\}
\end{aligned}\right.
$$

then drive system (20) will achieve projective synchronization with response system (21).

(ii) Suppose that $m_{1}=m_{2}=m_{3}=m_{4}=0$ and $k_{1}=k_{2}=k_{3}=k_{4}=1$, and if the controllers are chosen as follows:

$$
\left\{\begin{aligned}
U_{1}= & -\left\{\left(w_{1}-l_{1} u_{1}\right)+\left[\rho_{3}\left(w_{3}-w_{1}\right)+w_{6}-l_{1} \alpha_{1}\left(u_{3}-u_{1}\right)-l_{1} u_{6}\right]-\alpha_{1}\left(w_{2}-l_{1} u_{2}\right)\right\} \\
U_{2}= & -\left\{\left(w_{2}-l_{1} u_{2}\right)+\left[\rho_{3}\left(w_{4}-w_{2}\right)-l_{1} \alpha_{1}\left(u_{4}-u_{2}\right)\right]+\alpha_{1}\left(w_{1}-l_{1} u_{1}\right)-\alpha_{2}\left(w_{3}-l_{2} u_{3}\right)\right\} \\
U_{3}= & -\left\{\left(w_{3}-l_{2} u_{3}\right)+\left[\left(-w_{1} w_{5}+\nu_{3} w_{3}+w_{6}\right)-l_{2}\left(\gamma_{1} u_{1}-u_{3}-u_{1} u_{5}\right)\right]+\alpha_{2}\left(w_{2}-l_{1} u_{2}\right)\right. \\
& \left.-\beta_{1}\left(w_{4}-l_{2} u_{4}\right)\right\} \\
U_{4}= & -\left\{\left(w_{4}-l_{2} u_{4}\right)+\left[\left(-w_{2} w_{5}+\nu_{3} w_{4}\right)-l_{2}\left(\gamma_{1} u_{2}-u_{4}-u_{2} u_{5}\right)\right]+\beta_{1}\left(w_{3}-l_{2} u_{3}\right)-\beta_{2}\left(w_{5}-l_{3} u_{5}\right)\right\} \\
U_{5}= & -\left\{\left(w_{5}-l_{3} u_{5}\right)+\left[\left(w_{1} w_{3}+w_{2} w_{4}-\mu_{3} w_{5}\right)-l_{3}\left(u_{1} u_{3}+u_{2} u_{4}-\beta_{1} u_{5}+u_{6}\right)\right]+\beta_{2}\left(w_{4}-l_{2} u_{4}\right)\right. \\
& \left.-\gamma_{1}\left(w_{6}-l_{4} u_{6}\right)\right\} \\
U_{6}= & -\left\{\left(w_{6}-l_{4} u_{6}\right)+\left[\left(w_{1} w_{3}+w_{2} w_{4}-\sigma_{3} w_{6}\right)-l_{4}\left(u_{1} u_{3}+u_{2} u_{4}-\sigma_{1} u_{6}\right)\right]+\gamma_{1}\left(w_{5}-l_{3} u_{5}\right)\right\}
\end{aligned}\right.
$$

then drive system (19) will achieve projective synchronization with response system (21).

Corollary 4. Suppose that $l_{1}=l_{2}=l_{3}=l_{4}=0, m_{1}=m_{2}=m_{3}=m_{4}=0$ and $k_{1}=k_{2}=k_{3}=$ $k_{4}=1$, and if the controllers are chosen as follows:

$$
\left\{\begin{array}{l}
U_{1}=-\left\{w_{1}+\left[\rho_{3}\left(w_{3}-w_{1}\right)+w_{6}\right]-\alpha_{1} w_{2}\right\} \\
U_{2}=-\left[w_{2}+\rho_{3}\left(w_{4}-w_{2}\right)+\alpha_{1} w_{1}-\alpha_{2} w_{3}\right], \\
U_{3}=-\left[w_{3}+\left(-w_{1} w_{5}+\nu_{3} w_{3}+w_{6}\right)+\alpha_{2} w_{2}-\beta_{1} w_{4}\right], \\
U_{4}=-\left[w_{4}+\left(-w_{2} w_{5}+\nu_{3} w_{4}\right)+\beta_{1} w_{3}-\beta_{2} w_{5}\right], \\
U_{5}=-\left[w_{5}+\left(w_{1} w_{3}+w_{2} w_{4}-\mu_{3} w_{5}\right)+\beta_{2} w_{4}-\gamma_{1} w_{6}\right], \\
U_{6}=-\left[w_{6}+\left(w_{1} w_{3}+w_{2} w_{4}-\sigma_{3} w_{6}\right)+\gamma_{1} w_{5}\right],
\end{array}\right.
$$

then system (21) is stabilized to the equilibrium, $O(0,0,0,0,0,0)$.

In what follows, numerical experiments are given to demonstrate our results. The fourth-order Runge-Kutta method is used with a time step size of 0.001 . The system parameters are given as 
Figure 4. Combination synchronization errors, $e_{1}, e_{2}, e_{3}, e_{4}, e_{5}$ and $e_{6}$, between drive systems (19), (20) and response system (21).
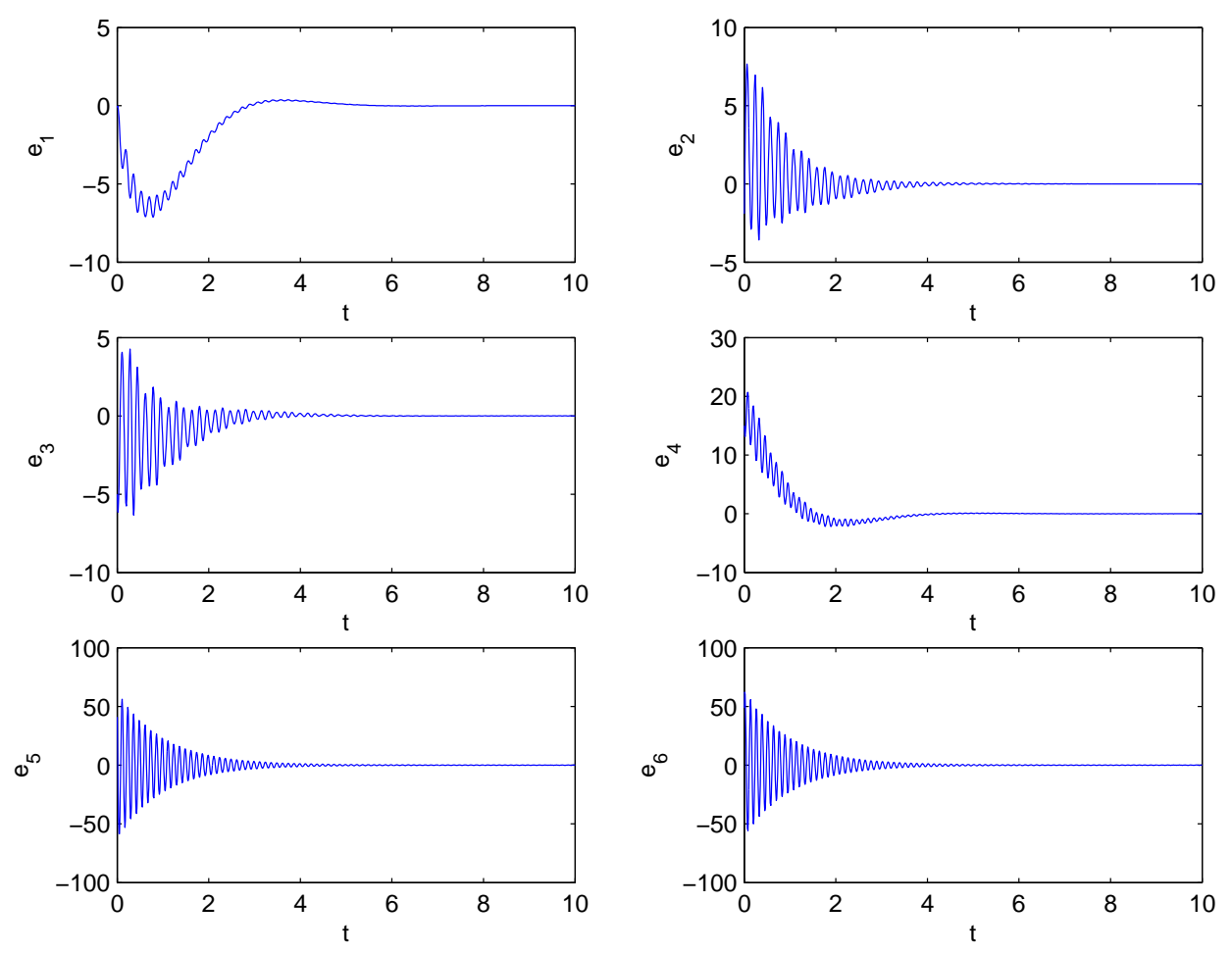

$\alpha_{1}=8, \beta_{1}=5, \gamma_{1}=50, \sigma_{1}=15, \alpha_{2}=36, \beta_{2}=4, \gamma_{2}=25, d_{2}=5, \rho_{3}=42, \nu_{3}=25, \mu_{3}=6$ and $\sigma_{3}=5$, so that the three complex nonlinear hyperchaotic systems exhibit hyperchaotic behaviors, respectively.

First, we assume $k_{1}=k_{2}=k_{3}=k_{4}=1, l_{1}=l_{2}=l_{3}=l_{4}=1$ and $m_{1}=m_{2}=m_{3}=m_{4}=1$, and the initial states for the drive systems and response systems are arbitrarily given by $\left(x_{1}(0), x_{2}(0), x_{3}(0), x_{4}(0)\right)=(2.0-i, 5.8-$ $2 i-12,-16),\left(y_{1}(0), y_{2}(0), y_{3}(0), y_{4}(0)\right)=(1.7+2.3 i, 0.1-14 i,-16,-18)$ and $\left(z_{1}(0), z_{2}(0), z_{3}(0), z_{4}(0)\right)=(3.6-0.6 i, 0.9-i, 13,15)$, i.e., $\left(u_{1}(0), u_{2}(0), u_{3}(0), u_{4}(0), u_{5}(0), u_{6}(0)\right)=$ $(2.0,-1,5.8,-2,-12,-16),\left(v_{1}(0), v_{2}(0), v_{3}(0), v_{4}(0), v_{5}(0), v_{6}(0)\right)=(1.7,2.3,0.1,-14,-16,-18)$ and $\left(w_{1}(0), w_{2}(0), w_{3}(0), w_{4}(0), w_{5}(0), w_{6}(0)\right)=(3.6,-0.6,0.9,-1,13,15)$, respectively. The corresponding numerical results are shown in Figures 4 and 5. Figure 4 displays the time response of the combination synchronization errors, $e_{1}, e_{2}, e_{3}, e_{4}, e_{5} a n d e_{6}$. The errors converge to zero, which implies that systems (19), (20) and (21) have achieved combination synchronization. Figure 5 depicts the time responses of the states, $u_{1}+v_{1}$ versus $w_{1}, u_{2}+v_{2}$ versus $w_{2}, u_{3}+v_{3}$ versus $w_{3}, u_{4}+v_{4}$ versus $w_{4}, u_{5}+v_{5}$ versus $w_{5}$ and $u_{6}+v_{6}$ versus $w_{6}$, respectively. When $k_{1}=k_{2}=k_{3}=k_{4}=1, l_{1}=l_{2}=l_{3}=l_{4}=0$ and $m_{1}=m_{2}=m_{3}=m_{4}=0$, the time evolution of the states, $w_{1}, w_{2}, w_{3}, w_{4}, w_{5}, w_{6}$, of system (21) with controller (29) are displayed in Figure 6, which means that system (21) is stabilized to the equilibrium, $O(0,0,0,0,0,0)$. 
Figure 5. Time responses for states $u_{i}+v_{i}$ versus $w_{i}, i=1,2, \ldots, 6$, respectively.
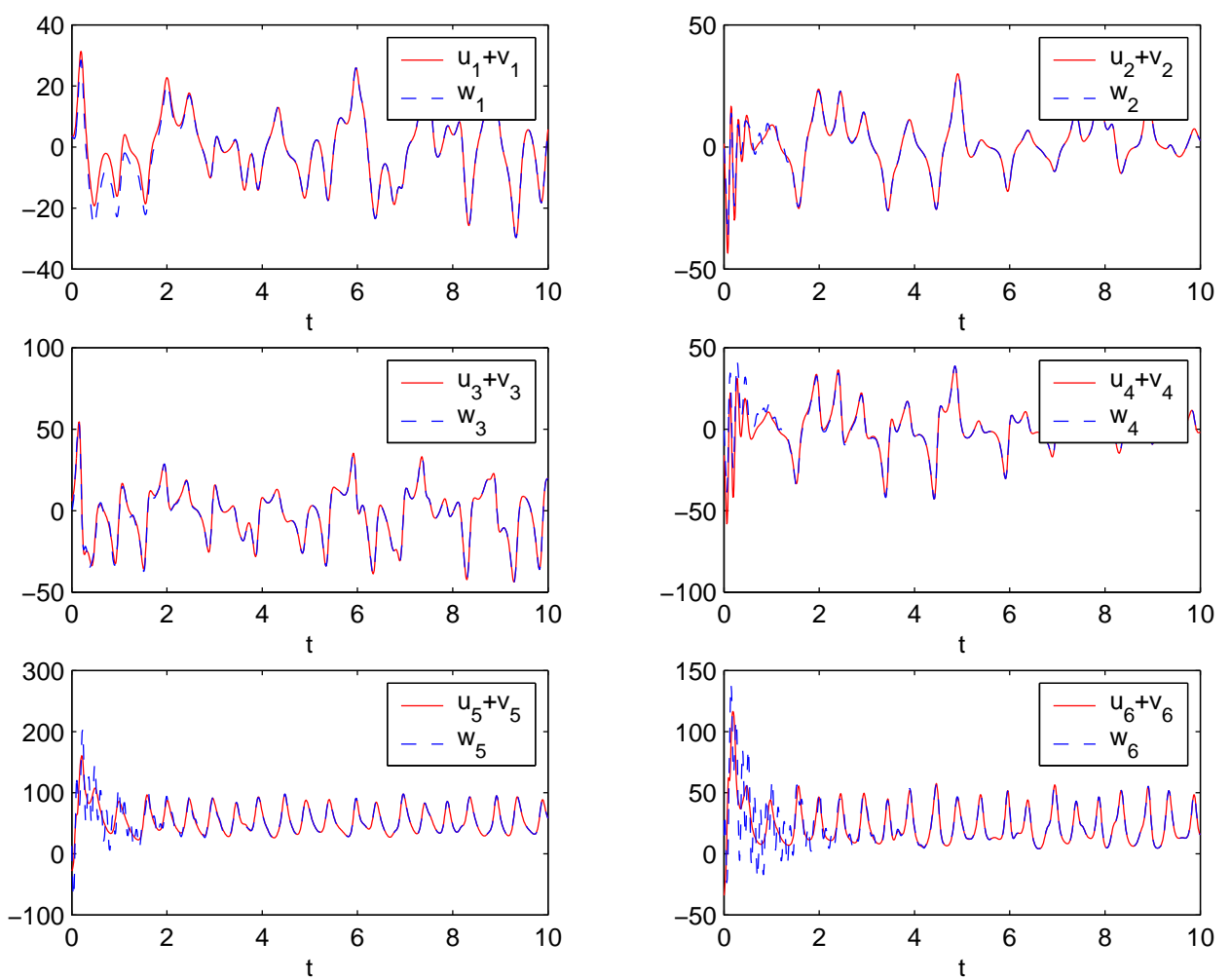

Figure 6. Time evolution of the states for system (21).
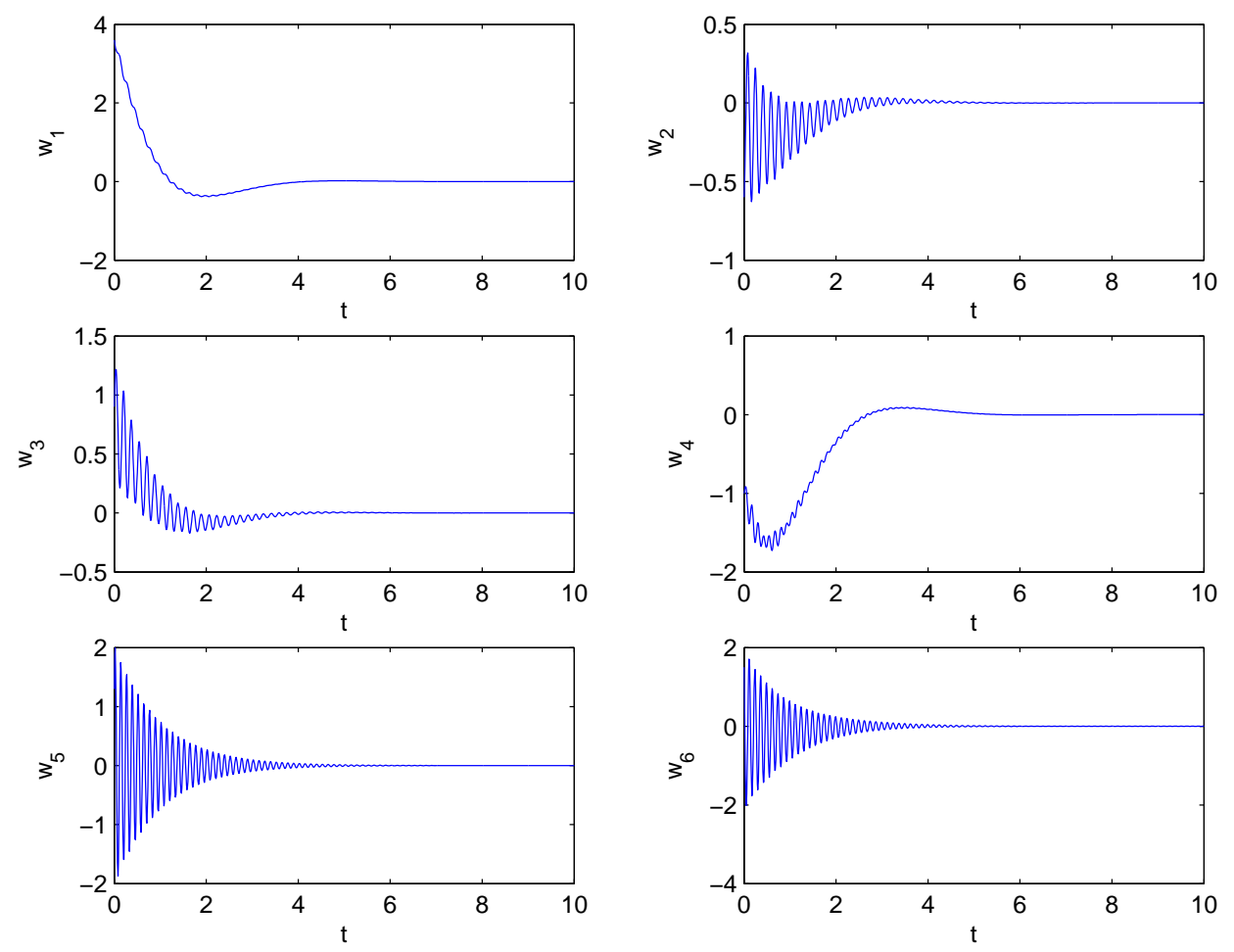


\section{Conclusions}

This paper investigates the combination synchronization of three nonlinear complex hyperchaotic systems: the complex hyperchaotic Lorenz system, the complex hyperchaotic Chen system and the complex hyperchaotic Lü system. Based on the Lyapunov stability theory, corresponding controllers to achieve combination synchronization among three identical or different nonlinear complex hyperchaotic systems are derived, respectively. Numerical simulations are conducted to illustrate the validity and feasibility of the theoretical analysis. When applying the complex systems in communications, the complex variables will double the number of variables and can increase the content and security of the transmitted information. Furthermore, combination synchronization between two drive systems and one response system has obvious advantages over synchronization between one drive system and one response system. Thus combination synchronization of complex nonlinear systems can find better applications in security communication. However, in practical chaotic synchronization, mismatched parameters exist, and the external disturbances are always unavoidable [17]. In our future work, we will investigate robust combination synchronization in the existence of mismatched parameters and external disturbances.

\section{Acknowledgments}

The authors sincerely thank the referees for their helpful comments. This work was supported by the the Natural Science Foundation of Yunnan Province under grant No. 2009CD019 and the Natural Science Foundation of China under grants No. 61065008 and No. 11161055.

\section{Conflicts of Interest}

The authors declare no conflict of interest.

\section{References}

1. Fowler, A.C.; Gibbon, J.D.; McGuinness, M.J. The complex Lorenz equations. Phys. D 1982, 4, 139-163.

2. Mahmoud, E.E. Dynamics and synchronization of new hyperchaotic complex Lorenz system. Math. Comput. Model. 2012, 55, 1951-1962.

3. Mahmoud, G.M.; Farghaly, A.A.M. Chaos control of chaotic limit cycles of real and complex van der Pol oscillators. Chaos Solitons Fractals 2004, 21, 915-924.

4. Mahmoud, G.M.; Bountis, T.; Mahmoud, E.E. Active control and global synchronization of the complex Chen and Lü systems. Int. J. Bifur. Chaos 2007, 17, 4295-4308.

5. Mahmoud, G.M.; Mahmoud, E.E.; Ahmed, M.E.A. hyperchaotic complex Chen system and its dynamics. Int. J. Appl. Math. Stat. 2007, 12, 90-100.

6. Mahmoud, G.M.; Ahmed, M.E.; Mahmoud, E.E. Analysis of hyperchaotic complex Lorenz systems. Int. J. Mod. Phys. C 2008, 19, 1477-1494.

7. Mahmoud, G.M.; Mahmoud, E.E.; Ahmed, M.E. On the hyperchaotic complex Lü system. Nonlinear Dyn. 2009, 58, 725-738. 
8. Mahmoud, G.M.; Al-Kashif, M.A.; Farghaly, A.A. Chaotic and hyperchaotic attractors of a complex nonlinear system. J. Phys. A Math. Theor. 2008, 41, e055104.

9. Mahmoud, G.M.; Ahmed, M.E.; Sabor, N. On autonomous and nonautonomous modified hyperchaotic complex Lü systems. Int. J. Bifur. Chaos 2011, 21, 1913-1926.

10. Pecora, L.M.; Carroll, T.L. Synchronization in chaotic systems. Phys. Rev. Lett. 1990, 64, 821-824.

11. Mahmoud, G.M.; Mahmoud, E.E. Complete synchronization of chaotic complex nonlinear systems with uncertain parameters. Nonlinear Dyn. 2010, 62, 875-882.

12. Mahmoud, G.M.; Mahmoud, E.E. Phase and antiphase synchronization of two identical hyperchaotic complex nonlinear systems. Nonlinear Dyn. 2010, 61, 141-152.

13. Mahmoud, G.M.; Mahmoud, E.E.; Arafa, A.A. On projective synchronization of hyperchaotic complex nonlinear systems based on passive theory for secure communications. Phys. Scr. 2013, 87, e055002.

14. Liu, P.; Liu, S.; Li, X. Adaptive modified function projective synchronization of general uncertain chaotic complex systems. Phys. Scr. 2012, 85, e035005.

15. Luo, R.Z.; Wang, Y.L.; Deng, S.C. Combination synchronization of three classic chaotic systems using active backstepping design. Chaos 2011, 21, e043114.

16. Luo, R.Z.; Wang, Y.L. Finite-time stochastic combination synchronization of three different chaotic systems and its application in secure communication. Chaos 2012, 22, e023109.

17. Ahn, C.K.; Jung, S.T.; Kang, S.K.; Joo, S.C. Adaptive $H \infty$ synchronization for uncertain chaotic systems with external disturbance. Commun. Nonlinear Sci. Numer. Simulat. 2010, 15, 2168-2177.

(c) 2013 by the authors; licensee MDPI, Basel, Switzerland. This article is an open access article distributed under the terms and conditions of the Creative Commons Attribution license (http://creativecommons.org/licenses/by/3.0/). 